\title{
$\therefore-$
}

NASA Technical Memorandum 102428

AIAA-90-0028

\section{Application of an Efficient Hybrid Scheme for Aeroelastic Analysis of Advanced Propellers}

R. Srivastava and N.L. Sankar

Georgia Institute of Technology

Atlanta, Georgia

T.S.R. Reddy

The University of Toledo

Toledo, Ohio

and

D.L. Huff

Lewis Research Center

Cleveland, Ohio

Prepared for the

28th Aerospace Sciences Meeting

2.... sponsored by the American Institute of Aeronautics and Astronautics

Reno, Nevada, January 8-11, 1990

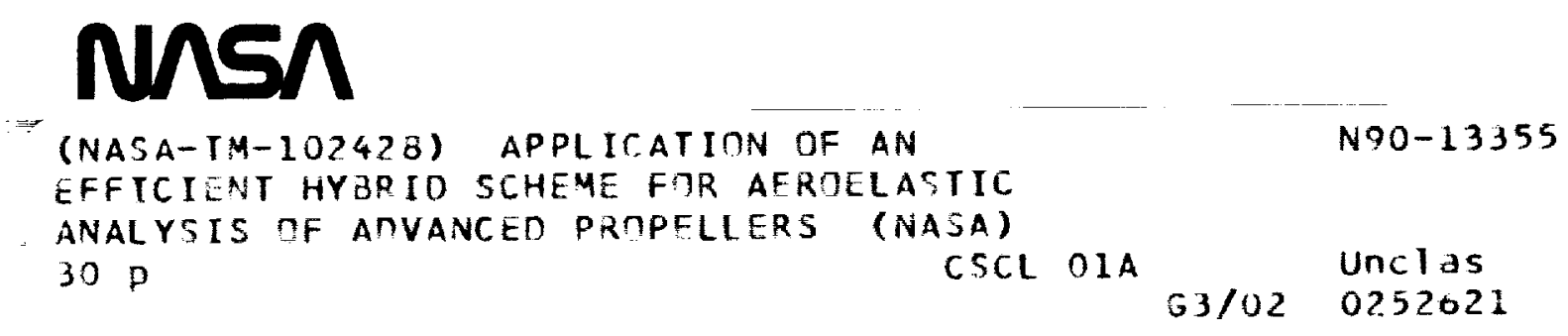




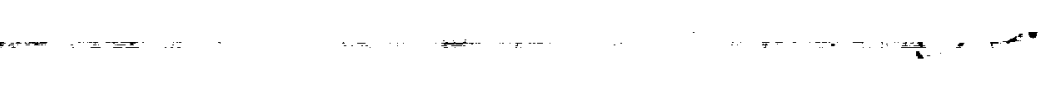

$=$ $+\cdots$

(1)

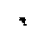

$=$

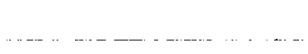




\title{
Application of an Efficient Hybrid Scheme for Aeroelastic Analysis of Advanced Propellers
}

\author{
R. Srivastava* and N.L. Sankar* \\ Georgia Institute of Technology \\ Atlanta, Georgia \\ T.S.R.Reddy $\dagger$ \\ The University of Toledo \\ Toledo, Ohio \\ and \\ D.L. Huff $† \dagger$ \\ National Aeronautics and Space Administration \\ Lewis Research Center \\ Cleveland, Ohio
}

\begin{abstract}
An efficient three-dimensional hybrid scheme is applied for solving Euler equations to analyze advanced propellers. The scheme treats the spanwise direction semi explicitly and the other two directions implicitly, without affecting the accuracy; as compared to a fully implicit scheme. This leads to a reduction in computer time and memory requirement.

The calculated power coefficients for two advanced propellers, SR3 and SRiL, and various advance ratios showed good correlation with experiment. Spanwise distribution of elemental power coefficient and steady pressure coefficient differences a!so showed good agreement with experiment. A study of the effect of structural flexibility on the performance of the advanced propellers showed that structural deformation due to centrifugal and aero loading should be included for better correlation.
\end{abstract}

*Member AIAA and AHS.

$\lceil$ NASA Resident Research Associate at Lewis Research Center; member AIAA and AHS. ††Member AIAA. 


\section{Introduction}

It has been known for some time now that the best propulsive efficiency is offered by propellers. However the efficiency drops off very rapidly as the cruise Mach number increases beyond 0.5 , as high tip Mach numbers lead to high compressibility losses (due to wave drag). Currently an effort is underway to improve the propulsive efficiency of commercial and military aircraft. Newly designed high speed advanced propellers, also known as propfans, show a very high propulsive efficiency at cruise speeds upto Mach $0.8[1]$.

The propfans are designed to delay the compressibility losses, thus extending the high efficiency of a propeller to relatively higher cruise Mach numbers. This is accomplished by sweeping the blade backwards and using thinner airfoils, on the outboard section of the blade. In addition low aspect ratio blades are used. This, combined with high tip Mach number, leads to high blade twist and high disk loading. The requirement of high disk loading further dictates a large number of blades per propeller, which must maintain structural integrity. However, these special features of the propfans lead to new problems.

One of the critical problems arises due to the fact that these blades are thin and hence moderately flexible. They are also highly swept near the tips, and hence are succeptible to transonic classical flutter or large amplitude oscillations. As concluded by Mehmed and Kaza [2], through wind tunnel tests of a propfan, there exists a strong aerodynamic coupling or cascade effect between blades. They also observed a classical bending - torsion unstalled flutter for a wide range of blade loading.

To understand and alleviate the problems associated with the propfan, the flow phenomena on the blades have to be accurately known. Also, in order to obtain the loads, an accurate prediction of pressure distribution on the blade is required. These could be obtained through experimental or numerical techniques. At the design stage experimental techniques are very expensive. Therefore, a need definitely exists to support the development of potentially high propulsive efficiency propfans through numerical techniques. With numerical techniques it is easier and cheaper to obtain pressure distribution, and flow details can easily be obtained at any point in the flow field. 
The existing numerical methods vary in complexity from simple Goldstein type strip analysis to analyses that solve the Euler and Navier - Stokes equations. The strip theory based on Goldstein's work [3], assumes the flow to be inviscid and incompressible (hence irrotational). The propeller is modeled by a lifting line vortex and the wake is assumed to be composed of a rigid helical vortex sheet. In this analysis the propeller is restricted to having straight blades and no provision can be made for the nacelle, since the vortex wake extends to the axis. Sullivan [4] has improved on this method by using the curved lifting line concept to account for the sweep. In this approach the vortex wake is represented by a finite number of vortex filamenis in place of the continuous sheet of vorticity as used in Goldstein's approach. The analysis has been further extended in reference [5] by placing the vortex filaments along the stream surfaces so that they conform to the shape of the axisymmetric nacelle.

Hanson [6] and Williams [7] applied the Kernel function approach to a propfan blade. They numerically solve a linear integral equation for upwash angle due to the blade pressure distribution by discretizing the load representation. The friction drag is obtained from the two- dimensional airfoil tables as a function of lift coefficient for the appropriate section camber, thickness and a Mach number adjusted for sweep and three-dimenzional effects. The induced drag is obtained by determining the kinetic energy-per-unit-length of the far wake. The methods mentioned so far are based on linearized analyses. However, as the advanced propeller operates at or near transonic tip Mach number, flow nonlinearities may become important.

Jou [8] has applied the finite volume approach of Jameson ? for the analysis of propfans using full potential equation. The formulation was not able to provide converged solutions for free stream Mach numbers greater than 0.6. It was concluded that strong rotational flow effects were present near the leading edge, which could not be modelled by the potential equation. In addition the potential flow equations at times, lead to non-unique solutions.

Chausee [10] and Whitfield et al. [11] have applied the unsteady, three dimensional Euler equations to the propfan geometry. Matsuo et al. [12] have recently solved the full Navier - Stokes equations around a propfan. Some of these methods have been reviewed in reference [13], with regards to performance prediction. 
All the analyses mentioned so far, with the exception of Whitfield et al. [11] have been for axisymmetric flows. For a propfan in flight configuration, the flow is not axisymmetric. Even for cruise conditions the nacelle is at an angle of attack to the free stream, which destroys the axisymmetric nature of the flow. A true unsteady analysis would permit the analysis of the propfan in all flight configurations, including climb and descent. The propfan may also encounter off design conditions such as gusts or cross winds due to the disturbances in atmosphere. The cross winds could result in the propfan being exposed to the wake of the fuselage. The performance of the propfan due to gusts or fuselage wake may be very critical for the safety of the aircraft, and it should be possible to include them in the analysis.

The primary objective of the present research is to develop a method to solve unsteady Euler equations to predict flowfield around a propfan. The solution method should be able to solve the unsteady flow field around a propfan in non-axisymmetric flight condition, undergoing time dependent forcing function, unsteady blade vibratory motion etc. To accomplish this objective, a versatile body fitted grid is used. The blade motion is simulated by grid motion, allowing any time dependent blade motion. This will permit the calculation of both forced and free response due to any time dependent forcing function for a flexible blade. It also allows the governing equations to be cast in Cartesian coordinates, and yet be able to simulate a rotating blade. A Cartesian grid system simplifies the governing equations, as the Coriolis forces do not appear explicitly. This is true not only for a rotating blade but also for a blade undergoing time dependent arbitrary motion.

The governing equations in fully conservative form are discretized using second order accurate central differencing for the spatial derivatives and a first order upwind differencing for the temporal derivative, to obtain a set of algebraic equations. The Alternating Direction Implicit (ADI) scheme is used to solve the algebraic equations. The geometry of the propfan and the spanwise load distribution permits the grid to be at least an order of magnitude larger in the spanwise direction as compared to the other two directions. This allows the radial direction fluxes to be treated semi - explicitly, and the other two directions implicitly, without affecting the accuracy 
significantly as compared to a fully implicit scheme. Treating one direction semiexplicitly requires only two costly inversions of block tridiagonal matrix, as opposed to three inversions for a fully implicit scheme, per time step. It also reduces the memory requirement as only two time levels of information needs to be stored at any given time, one of which needs to be only two dimensional. The use of such a hybrid scheme leading to reduction in computer time and memory requirement makes the scheme more efficient.

The specific objectives of the present paper are 1) to apply an efficient hybrid scheme to analyze advanced propellers, 2) to calculate steady performance, 3) to include structural deformation, due to centrifugal and steady aero loading in the analysis, 4) to study the effects of structural flexibility on the performance of advanced propellers. The governing equations and the numerical solution method are described first, followed by results and discussion. The methods developed here are expected to be helpful for future aeroelastic research.

\section{Formulation}

Aerodynamic Model:

The Euler equations, in conservation form and in Cartesian coordinates, can be written as:

$$
(\hat{\mathrm{q}})_{t}+(\hat{\mathrm{E}})_{x}+(\hat{\mathrm{F}})_{y}+(\hat{\mathrm{G}})_{z}=0
$$

where $\hat{\mathrm{q}}$ is the vector containing conserved flow properties. $\hat{\mathrm{E}}, \hat{\mathrm{F}}$ and $\hat{\mathrm{G}}$ are the nonlinear flux vectors which are functions of the vector $\hat{\mathrm{q}}$. The subscripts denote the partial derivative of the vector. In the above equation

$$
\hat{\mathrm{q}}=\left(\begin{array}{c}
\rho \\
\rho u \\
\rho v \\
\rho w \\
e
\end{array}\right) \quad \hat{\mathrm{E}}=\left(\begin{array}{c}
\rho u \\
\rho u^{2}+p \\
\rho u v \\
\rho u w \\
u(e+p)
\end{array}\right)
$$




$$
\hat{\mathbf{F}}=\left(\begin{array}{c}
\rho v \\
\rho u v \\
\rho v^{2}+p \\
\rho v w \\
v(e+p)
\end{array}\right) \quad \hat{\mathrm{G}}=\left(\begin{array}{c}
\rho w \\
\rho w w \\
\rho v w \\
\rho w^{2}+p \\
w(e+p)
\end{array}\right)
$$

where $\rho$ is the fluid density, $u, v, w$ are the inertial Cartesian components of the flow velocity, $e$ is the total energy of the fluid per unit volume and $p$ is the hydrodynamic pressure and may be expressed using the equation of state for perfect gas as:

$$
p=(\gamma-1)\left[e-\frac{1}{2} \rho\left(u^{2}+v^{2}+w^{2}\right)\right]
$$

where $\gamma$ is the ratio of specific heats. The advantage of using the conservation form is that it ensures the conservation of physical flux properties across discontinuities (e.g. shock) in the flow [14].

In order to analyze flow past an arbitrary geometry undergoing arbitrary motion, these equations need to be transformed and recast in a generalized coordinate system. The coordinates of the generalized system, have the following one to one relationship with the coordinates in the physical domain of interest :

$$
\begin{aligned}
\xi & =\xi(x, y, z, t) \\
\eta & =\eta(x, y, z, t) \\
\zeta & =\zeta(x, y, z, t) \\
\tau & =t
\end{aligned}
$$

These coordinates are non orthogonal and completely general. The equation (1) can be rewritten as: 


$$
\mathbf{q}_{\tau}+\mathrm{E}_{\xi}+\mathrm{F}_{\eta}+\mathrm{G}_{\zeta}=0
$$

where

$$
\begin{gathered}
\mathbf{q}=J^{-1}\left(\begin{array}{c}
\rho \\
\rho u \\
\rho v \\
\rho w \\
e
\end{array}\right) \quad \mathrm{E}=J^{-1}\left(\begin{array}{c}
\rho U \\
\rho u U+\xi_{x} p \\
\rho v U+\xi_{y} p \\
\rho w U+\xi_{x} p \\
(e+p) U-\xi_{t} p
\end{array}\right) \\
\mathbf{F}=J^{-1}\left(\begin{array}{c}
\rho \\
\rho v V+\eta_{y} p \\
\rho w V+\eta_{z} p \\
(e+p) V-\eta_{t} p
\end{array}\right) \quad \mathrm{G}=J^{-1}\left(\begin{array}{c}
\rho W \\
\rho u W+\zeta_{x} p \\
\rho v W+\zeta_{y} p \\
\rho w W+\zeta_{z} p \\
(e+p) W-\zeta_{t} p
\end{array}\right)
\end{gathered}
$$

$U, V$, and $W$ are the contravariant velocities, and $J$ is the jacobian and $\xi_{x}, \eta_{x}, \zeta_{x}$ etc. are the metrics of transformation.

\section{Initial and Boundary Conditions}

A large number of problems can be described by the same set of governing equations. It is the proper application of the boundary condition that makes the solution unique to any given problem. Hence using proper and physically meaningful boundary conditions is as important as the correct governing equations.

The initial conditions may be critical to convergence of the numerical scheme. An intelligent guess of the initial conditions could help in achieving convergence faster. For these calculations the free stream conditions are used as the initial condition. 
In the present analysis the flow variables at the boundaries are updated explicitly after the governing equations have been solved for the interior flow field. The following boundary conditions need to be addressed:

The blade and nacelle - surface boundary condition :

Physically, there can be no flow through or on a solid surface, hence the velocity on a solid surface must go to zero. The physical boundary condition of no slip can be ignored for the Euler equations. Thus the boundary condition on the blade and nacelle surfaces can be mathematically written as:

$$
\overrightarrow{V_{b}} \cdot \vec{n}=0
$$

Where $\vec{V}_{b}$ is the velocity vector at the surface and $\vec{n}$ is the unit vector normal to the surface. The velocity vector $\vec{V}_{b}$, at any point $(x, y, z)$ in the blade fired coordinate can be given as

$$
\vec{V}_{b}=\left(u-x_{\tau}\right) \hat{i}+\left(v-y_{\tau}\right) \hat{j}+\left(w-z_{\tau}\right) \hat{k}
$$

\section{Far-field conditions:}

Since the propfan is operating in free air, the far field conditions should be the same as that of the free air. For steady state calculations all disturbances from the solid surface must propagate to infinity. On the subsonic inflow bouncary, one characteristic should be allowed to escape hence $\rho$ is extrapolated and the remaining variables $\rho u, \rho v, \rho w$ and $e$ are fixed at the free stream value. For a supersonic inflow boundary, all quantities are fixed to that of the free stream,as disturjances cannot travel upstream in a supersonic flow. At the subsonic outflow boundary, four characteristics should escape, thus the four quantities $\rho, \rho u, \rho v, \rho w$ are extrapolated from inside while the pressure is fixed to that of the free stream. For supessonic outflow, all characteristics should escape, hence all quantities are extrapolated i:om inside the flow domain. 
The block interface boundary:

It is neither efficient nor practical to solve all the blades at the same time, hence, one blade passage is handled at a time. This introduces additional boundaries for computation. Across these boundaries all the variables must be continuous, except on solid boundaries and boundaries downstream of the blade. The boundary condition, for these boundaries, depends on the type of flow being solved. An axisymmetric flow would require periodicity on the fluid interface boundaries. Periodicity will require that the two boundaries have same fluid properties. As shown in figure (1a), the fluid properties at the boundaries $\mathrm{K}=1$ and $\mathrm{K}=\mathrm{KMAX}$ are updated as the average of fluid properties at $\mathrm{K}=2$ and $\mathrm{K}=\mathrm{KMAX}-1$ for a symmetric flow field.

For an unsymmetric flow, the periodicity on these boundaries does not exist. Therefore, in order to obtain the solution for such a case, the whole propfan should be solved. This is done by advancing the solution of each block one time step, one block at a time. In this case again the boundaries are updated explicitly, after the interior points have been updated.This is done by averaging the flow variables from the nodes on each side of the boundary from the adjoining blocks. Referring to figure (1b), (the subscripts refer to the corresponding block) the quantities at boundary $\mathrm{K}=\mathrm{KMAX}$ of block $\mathrm{N}$ (which is also the boundary $\mathrm{K}=1$ for block $\mathrm{N}+1$ ) would be the average of flow quantities at $K=K M A X-1$ of block $N$ and $K=2$ of block $N+1$. In doing so the latest available values at any given time are used.

\section{Solution Procedure:}

The descretized forms of the governing equations described earlier, are solved using a hybrid scheme, described in next section. The algebraic equations are approximately factorized and solved using the ADI scheme. An implicit method is more demanding as far as coding is concerned. However it allows larger time steps to be taken as opposed to the explicit schemes.

Time integration is carried out using the first order accurate Euler implicit rule

$$
\mathrm{q}^{n+1}=\mathrm{q}^{n}+\Delta \tau \frac{\partial \mathbf{q}^{n+1}}{\partial \tau}
$$


where the superscript $n$ denotes the current time level, at which the flow variables are known, and $n+1$ the next or unknown time level. Even though this is a first order accurate scheme, satisfactory time accuracy is obtained because a relatively small time step is required to maintain numerical stability.

Substituting the Euler equations (5) in (9) we get

$$
\mathrm{q}^{n+1}=\mathrm{q}^{\mathrm{n}}-\Delta \tau\left(\mathrm{E}_{\xi}+\mathrm{F}_{\eta}+\mathrm{G}_{\zeta}\right)^{n+1}
$$

The partial derivatives $E_{\xi}, F_{\eta}, G_{\zeta}$ are obtained using the standard second order accurate central differences.

The hybrid scheme:

In order to decrease the computational time, flux terms in two directions $(\xi, \zeta)$, are treated implicitly while the radial direction $(\eta)$ flux terms are treated semi-explicitly. The $\eta$ derivative is obtained using the latest available values of the flow variables. This method requires only two costly inversions of the block tridiagonal matrix, in the two implicit directions. Rizk and Chausee [15] first used this hybrid scheme with the Beam and Warming algorithm. Using this technique the solver marches along the $\eta$ direction, solving the equations one $\eta$ plane at a time. The marching direction is reversed after every sweep, in order to remove any dependency on the marching direction. Equation (10) can then be rewritten as:

$$
\mathrm{q}^{n+1}=\mathrm{q}^{n}-\Delta \tau\left(\mathrm{E}_{\xi}^{n+1}+\mathrm{F}_{\eta}^{n, n+1}+\mathrm{G}_{\zeta}^{n+1}\right)
$$

Since the $\eta$ marching direction is changed every iteration, the $\mathrm{F}_{\eta}^{n, n+1}$ alternates between

$$
\frac{F_{i, j+1, k}^{n}-F_{i, j-1, k}^{n+1}}{2 \Delta \eta}
$$


during the odd time steps, and

$$
\frac{F_{i, j+1, k}^{n+1}-F_{i, j-1, k}^{n}}{2 \Delta \eta}
$$

during the even time steps.

The above discretization leads to a set of algebraic equations for $\mathbf{q}$. These equations are costly to solve since the flux vectors $E$ and $G$ are highly nonlinear. The nonlinearity is removed by linearising the fluxes about the previous time step value, resulting in the following linear equation :

$$
\left[I+\Delta \tau\left(\delta_{\xi} A^{n}+\delta_{\zeta} B^{n}\right)\right] \mathrm{q}^{n+1}=\left[I+\Delta \tau\left(\delta_{\xi} A^{n}+\delta_{\zeta} B^{n}\right)\right] \mathrm{q}^{n}+\mathrm{R}^{n, n+1}
$$

where

$$
\mathrm{R}^{n, n+1}=-\Delta \tau\left(\delta_{\xi} \mathrm{E}^{n}+\delta_{n} \mathrm{~F}^{n, n+1}+\delta_{\zeta} \mathrm{G}^{n}\right)
$$

and the operator notation $\delta_{\xi}(A \mathbf{q})=\left[\delta_{\xi} A\right] \mathbf{q}$ and $\delta_{\zeta}(B \mathbf{q})=\left[\delta_{\zeta} B\right] \mathbf{q}$ is used.

This Euler equation formulation can be very easily extended to solve the NavierStokes equations by simply adding the viscous terms to the right hand side. This does not alter the numerical formulation.

Now defining $\Delta \mathrm{q}^{n+1}=\mathrm{q}^{n+1}-\mathrm{q}^{n}$, we can rewrite the equation (12) as

$$
\left[I+\Delta \tau\left(\delta_{\xi} A^{n}+\delta_{\zeta} B^{n}\right)\right] \Delta \mathbf{q}^{n+1}=\mathbf{R}^{n, n+1}
$$

Only two levels of storage, $q$ and $\Delta q$, are required, and since one direction is treated explicitly, the $\Delta \mathrm{q}$ array and the residual array $\mathrm{R}^{n, n+1}$ need to be only two dimensional. It is also possible to store $\Delta \mathrm{q}$ and $\mathrm{R}^{n, n+1}$ in the same memory locations, further reducing the memory requirements.

Even though the governing equation has been linearized, equation (14) is still very difficult to solve, as the matrix operator on the left hand side is very large and very sparse. However the matrix operator can be approximately factorized as 


$$
\left[I+\Delta \tau\left(\delta_{\xi} A+\delta_{\zeta} B\right)\right]=\left[I+\Delta \tau \delta_{\xi} A\right]\left[I+\Delta \tau \delta_{\zeta} B\right]+\bigcirc\left(\Delta \tau^{2}\right)
$$

This factorization does not affect the temporal accuracy. Equation (14) can then be written as

$$
\left[I+\Delta \tau \delta_{\xi} A^{n}\right]\left[I+\Delta \tau \delta_{\zeta} B^{n}\right]\left\{\Delta \mathbf{q}^{n+1}\right\}=\mathbf{R}^{n, n+1}
$$

or defining

$$
\Delta \mathrm{q}^{\wedge n+1}=\left[I+\Delta \tau \delta_{\zeta} B^{n}\right] \Delta \mathbf{q}^{n+1}
$$

we get the following system of equation:

$$
\begin{aligned}
{\left[I+\Delta \tau \delta_{\xi} A^{n}\right] \Delta \mathrm{q}^{\wedge n+1} } & =\mathrm{R}^{n, n+1} \\
{\left[I+\Delta \tau \delta_{\zeta} B^{n}\right] \Delta \mathrm{q}^{n+1} } & =\Delta \mathrm{q}^{\times n+1}
\end{aligned}
$$

These equations can be easily solved for $\Delta \hat{\mathbf{q}}^{n+1}$ by performing two successive block tridiagonal inversions. Since the $\xi$ and $\zeta$ directions are uncoupled, the two inversions are performed first with a $\xi$ sweep and then with a $\zeta$-sweep, thus the name 'Alternating Direction'. These inversions are performed at each spanwise station, marching along the spanwise direction. As mentioned earlier, the marching direction is reversed every iteration. Each element of the block tridiagonal matrix has $5 \times 5$ elements.

\section{Artificial Dissipation:}

The use of central difference, makes the scheme mildly unstable, and also introduces odd even decoupling. This is remedied by adding artificial dissipation. The implementation of artificial dissipation, in the present work is based on the formulation of Jameson et al. [16]. This scheme has a second order implicit dissipation and a blend of second/ fourth difference explicit dissipation terms. A scaling factor for both 
implicit and explicit dissipation is employed to control the amount of dissipation in the scheme. Adding the dissipation terms, equations (18) and (19) can be written as:

$$
\begin{aligned}
{\left[I+\Delta \tau\left(\delta_{\xi} A^{n}+\epsilon_{I} D_{I_{\xi}}\right)\right] \Delta \mathbf{q}^{\alpha n+1} } & =\mathbf{R}^{n, n+1}-\epsilon_{E} D_{E} \Delta \tau \\
{\left[I+\Delta \tau\left(\delta_{\zeta} B^{n}+\epsilon_{I} D_{I_{\zeta}}\right)\right] \Delta \mathbf{q}^{n+1} } & =\Delta \mathbf{q}^{\times n+1}
\end{aligned}
$$

where $D_{I_{\xi}}$ and $D_{I_{\zeta}}$ are second order implicit dissipation terms and $D_{E}$ is the explicit dissipation term, given in reference [22]. $\epsilon_{I}$ and $\epsilon_{E}$ are user supplied constants, which depend on grid spacing. At the boundaries the fourth order differences are repalced by second order differences.

\section{Aeroelastic Model:}

As mentioned earlier, the propfan has thin, swept, and twisted blades. Since the blades are thin and flexible, deflections due to centrifugal and steady aero loads are large. Hence, the aeroelastic problem is inherently nonlinear, requiring geometric nonlinear theory of elasticity [17].

The blades have large sweep and twist, which couples blade bending and torsional motions. They behave like plate-like structures because of the low aspect ratio. These factors require a finite element structural model which accounts for centrifugal softening and stiffening effects and, possibly, Coriolis effects. It has been found that the Coriolis effects are negligible for thin blades [18]. The centrifugal terms are important because of large blade sweep and flexibility. By assuming a rigid hub, the blades are structurally decoupled from one another. Consequently, it is sufficient to structurally model just one blade. Then, the governing aeroelastic equation can be written as

$$
\left\{\left[\mathbf{K}(\Omega)_{s}\right]+[\mathrm{K}(u)]\right\}\{u\}=\{\mathbf{P}(u)\}
$$

where $\left[K(\Omega)_{s}\right]$ is the centrifugal softening matrix, which is a function of the rotational speed, $\Omega,[\mathrm{K}(u)]$ is the nonlinear stiffness matrix which is a function of the 
nodal displacements, $\{u\}$ represents the blade deflections at the finite element nodes, and $\{\mathbf{P}(u)\}$ is the equivalent aerodynamic force vector.

In the present paper, the geometric nonlinear analysis was perfomed using the MSC/NASTRAN [19], in which the geometric nonlinear analysis is identified by " solution 64". The Solution 64 uses a modified Newton-Raphson algorithm, along with load updating to simulate the correct displacement-load relationship. It has the capability to update the displacement dependent centrifuga forces. The solution sequence is controlled through "subcases" or iterations with a minimum of two being required. The first subcase computes the initial, linear deflected shape. Subsequent subcases, then use the previously deflected shape to compute the differential stiffness matrix along with the new set of displacements [20].

The iterative method of solving equation (23) is shown in figure (2). Basically, a centrifugally deformed geometry is used to calculate steady aero loads with the Euler solver described in the previous section. These aero loads are then used to calculate a new deformed shape due to combined centrifugal and steady aero loads. The process, steps 4-6, is repeated until a converged, deformed geometry is obtained i.e., until the change in deflection from the $(i+1)^{\text {th }}$ iteration is equal to that from the $i^{\text {th }}$ iteration.

\section{Results and Discussion}

The hybrid numerical scheme discussed in the previous section, was first applied to an isolated aircraft wing in reference [21] and to a helicopter rotor blade in reference [22]. Typical results showing blade loading, are reproduced in figures (3) and (4).

As can be seen from both these figures, the hybrid scheme is able to predict flow phenomena of varying complexity with fairly good degree of accuracy.

The propfan blade has a much more complex shape than the aircraft wing or the helicopter blade. The high twist, large sweep, low aspect ratio, close proximity of other blades, presence of nacelle and thinner blades near the tip, make the flow field around it very complex. In the following, the flow solutions obtained for two advanced propellers, namely SR3 and SR7L, are presented. The calculations have been 
performed on a 'hot shape', obtained by including the deflections due to centrifugal loading in the undeflected blade shape ('cold shape').

A body fitted H-O grid was used for these calculations. A typical grid used in the calculation is shown in figure (5). The domain of calculation was taken to be the region between two blades with upper surface of one blade and lower surface of the adjoining blade as the boundaries of the domain. This region is referred to as blade passage. In general, in order to model the influence of adjacent blades (cascade effect) the entire propfan with all the blades (passages) are solved. However, for an axisymmetric flow field, considered here, all blade passages can be assumed to be identical, and only one blade passage is solved enforcing the conditions of symmetry. SR3 Propfan

The hybrid scheme, described earlier, was used to solve the flow field around an 8 bladed SR3 propfan. The SR3 propfan was designed to operate at a free stream Mach number of 0.8 , advance ratio of of 3.06 , at an altitude of 30,000 feet. Experimental data has been reported in [23], and the results obtained from the present analysis are compared in figures (6) and (7).

Figure (6) shows a comparison of the power coefficient of the propfan as a function of the advance ratio, with experimental [23] data. It also shows the comparison with other published numerical results $[11,12]$. As can be seen the comparison with experiment is good, however the power coefficients are consistently overpredicted. The results compare well with other published results, as well. As shown in reference [23] the power coefficients are quite sensitive to the blade setting angle, however an accurate measure of the blade setting angle, $\beta$, is difficult. Also, since the blades are thin and somewhat flexible, they are susceptible to deformations under loading during operation. These deformations result in a modified twist distribution on the blade. Also, the viscous effects are not included in the present analysis. Any or all of these phenomena could contribute to the overprediction of the power coefficient. 
The variation of elemental power coefficient with radial location is plotted and compared with experimental results in figure (7). In order to obtain a more meaningful comparison, the flight conditions were modified slightly in the numerical calculation, so as to match the experimental power coefficient. The power coefficient measured in experiment was 1.385 for a free stream Mach number of $0.8, \beta$ setting angle of $60.7 \mathrm{deg}$ and the advance ratio of 3.002 . The calculations were carried out with the free stream Mach number of 0.8 , the setting angle of $58.5 \mathrm{deg}$ and the advance ratio of 3.002 to obtain the same power coefficient. As can be seen a fairly good correlation is obtained, however, the elemental power is underpredicted for the inboard stations and overpredicted for the outboard stations. This is because the effect of the tip vortex is not properly accounted for. In these calculations no wake modelling is included, also the grid is not fine enough to properly capture the strength of the tip vortex. Capturing a weaker tip vortex results in smaller downwash velocity near the tip region, thus resulting in an over prediction ob blade loading near the tip. As the total power is matched with the experiment, an overprediction in the tip region results in an under prediction on the inboard region.

\section{SR7L Propfan}

The SR7L propfan has been designed for an operating free stream Mach number of 0.8 , rotational speed of $1700 \mathrm{rpm}$, at an altitude of 35,000 feet. In this section calculations for a two bladed SR7L propfan are presented. The aerodynamic calculations are first performed on the 'hot shape'. The effect of blade flexibility is then included in the calculations.

In figure (8) the elemental pressure coefficient difference is compared with experiment for a 2-bladed SR7L propfan. The blade was operating at a free stream Mach number of 0.775 and advance ratio of 3.088 . The $75 \%$ span setting angle was adjusted to match the power coefficient by a rigid body rotation of the blade about the pitch change axis. The pressure coefficient difference $\Delta C_{p}\left(C_{p_{l}}-C_{p_{u}}\right)$ is plotted and compared against experimental data [27] at various span locations. The comparison is good, except near the leading edge on the outboard stations. 
The effect of blade flexibility on performance was studied next for the SR7L propfan blade. The effect of flexibility is included by the aeroelastic iteration process, described earlier and shown in figure (2).

It is important that the blade finite element model accurately reflects its structural characteristics, since the entire analysis process is centered around the stiffness matrix. The NASTRAN finite element model used in this study is based on the final blade design [24]. The SR7L blade has an aluminum spar, nickel sheath, and fiber glass shell with foam fill. The shell, adhesive, spar, and shell filler material were combined using the Composite Blade Structural Analysis (COBSTRAN) program to produce equivalent, monolithic shell elements [25]. The finite element model of the SR7L blade is shown in figure (9a). The model has 261 nodes and 449 triangular shell elements. Bar elements are used to model the shank. Multipoint constraint grid chords are used to define the shank/blade interface [26].

The validity of the above finite element blade model has been shown in reference [20]. Frequencies and corresponding mode shapes were calculated over a range of speeds for a blade setting angle of 58 degrees and compared with those of Ref.[24]. For the sake of completeness, the calculated frequencies and the experimental values are reproduced in figure $(9 \mathrm{~b})$. The model showed good agreement at the first, third and fourth modes. The calculated second mode is much stiffer. This is a edgewise mode, which is the most sensitive mode to the support stiffness used in the finite element model. This mode is very difficult to model accurately [20], over a range of speeds because of nonlinear rotational effects. However, generally good agreement with the other modes implies an accurate determination of the blade's stiffness matrix, validating its use to calculate steady state deflections.

The aero loads obtained from the Euler solver are transferred to the NASTRAN grid for structural deformation calculations. The loads required as inputs to NASTRAN are the pressure differences at the centroids of the triangular element. As the 
two grids are not identical, an interpolation of the data was required. A spline interpolation was used to obtain the loads at the centroids of the elements. The deflections obtained from NASTRAN, under these loads, are then used to define the new blade shape. The loads are then recalculated for this new blade shape, and the process is repeated till convergence. The effect of the aeroelastic iteration on the calculation is shown in figures (10) through (14).

Figure ( 10 ) shows the $75 \%$ span twist or blade setting angle versus the iteration. The effect of centrifugal loads is seen as a change in the blade setting angle from iteration 0 to iteration 1. It shows that the centrifugal loads reduce the blade setting angle. This seems to be the largest effect on blade shape. Adding the deflections from centrifugal loads, to the blade shape gives the blade shape known as 'hot shape'. The loads obtained from this shape are then again used to obtain the new deflected shape. This iteration has been continued till the change in power coefficient is minimal. It shows that four iterations are sufficient for convergence to the final blade shape.

In figure (11) the thrust coefficient is plotted against the power coefficient for subsequent iterations. The setting angle used in the calculations has been obtained by rigid body rotation of the hot shape so as to match the power coefficient obtained by experiments. The experimental point is also plotted. The power coefficient obtained from the hot shape (marked 1), compares well with the experiment. However, the power coefficient changes considerably (marked 2 to 4 ), as the blade is allowed to deform under this load. It can be seen from figures (10) and (11), that the initial change in shape, lead to large change in power coefficient. For this particular case, under which the blades are loaded heavily almost $40 \%$ change in power coefficient is observed when the effect of aerodynamic loading is included in the blade shape. The subsequent changes are not as large. Hence, in order to obtain a better comparison with experimental power coefficient and load distribution on blade, the blade setting angle should be chosen such that the converged shape power coefficient is compared against the experimental data. This requires some trial and error in selecting the 'cold' or 'hot' shape from which the aeroelastic iteration should be started. Arriving 
at the final blade shape might be critical for vibration and flutter calculations, as well.

In figure(12) the relative change in twist angle over the span is plotted. This shows that the largest deflection occurs near the tip, with practically no deflection on the root sections. Also it should be noted that the variation in the blade twist is nonlinear and is largest near the tip. A rigid body rotation of the blade to account for the change in twist, would result in a linear variation along the span. This clearly shows that better performance calculations structural flexibility should be included in the analysis.

Figure (13) shows the in-plane deflection of the blade planform and figure (14) shows the out of plane deflection of the blade at constant chord. Again, the largest deflection is towards the tip, with practically no deflection towards the root. Figure (12) through (14) show clearly, that rigidly rotating the blade to match the power coefficient, does not simulate the correct blade shape. Also, as seen from figure (11) for highly loaded blades, these small differences might change the loading considerably. Computer Requirements:

All of the above computations were performed on the CRAY XMP24 computer available at NASA Lewis Research Center. For a grid size of $70 \times 35 \times 27$, the total memory and CPU time per time step required were $1.8 \mathrm{MW}$ and $2.4 \mathrm{sec}$. respectively. The NASTRAN run required $100 \mathrm{cpu}$ sec for 261 nodes, for each structural integration.

\section{Concluding Remarks}

This study showed that the hybrid scheme can be applied successfully to a propfan configuration (low aspect ratio, highly swept and very thin blades). In the numerical scheme described, the unsteady, time averaged Euler equations are solved in a fully conservative form. In the hybrid scheme two directions are treated implicitly and the spanwise direction is treated semi-explicitly. This reduces the computer time as only two costly inversions of the block tridiagonal matrix are required, corresponding to 
the two directions that are treated implicitly. It also reduces the computer memory requirement, as only one level of information need to be stored at any given time, thus making it a more efficient scheme.

From the present study following conclusions are drawn:

1. The calculated power coefficient for SR3 showed good correlation with experiment

2. The elemental power coefficient variation with radius compared well with experiment

3. The pressure coefficient difference for SR7L agreed well with measured values

4. The effect of aero loads was to compensate for the untwisting due to centrifugal loads

\section{Acknowledgements}

The authors wish to acknowledge Dr. Richard August of Sverdrup Technology Inc., Cleveland, OH, for many helpful suggestions. This work has been carried out under NASA grant NAG3-730 from NASA Lewis Research Center in Cleveland, Ohio, Mr. George L. Stefko, grant monitor.

\section{References}

[1] Whitlow, J. B., Jr. and Sievers, G. K.," Fuel Savings Potential of the NASA ATP," NASA TM 83736, 1984.

[2] Mehmed, O. and Kaza, K. R. V., "Experimental Classical Flutter Results of a Composite Advanced Turboprop Model," NASA TM-88792

[3] Goldstein, S.," On the Vortex Theory of Screw Propellers," Royal Society Proceedings, Vol. 123, No. 792, Apr. 6, 1929, pp 440-465 
[4] Sullivan, J. P.," The effect of Blade Sweep on Propeller Performance,"AIAA Paper 77-176, June 1977

[5] Egolf, T. A., Anderson, O. L., Edwards, D. E., and Landgrebe, A. J.," An Analysis for High Speed Propeller-Nacelle Aerodynamic Performance Prediction; Volume 1, Theory and Initial Application and Volume 2, User's Manual for the Computer Program," United Technologies Research Center, R79-912949-19, June 1979.

[6] Hanson, D. B.," Compressible Lifting Surface Theory for Propeller Performance Calculation," AIAA paper 82-0020.

[7] Williams, M. H., and Hwang, C.," Three Dimensional Unsteady Aerodynamics and Aeroelastic Response of Advanced Turboprops," AIAA Paper $86-0846$.

[8] Jou, W. H.," Finite Volume Calculation of the Three - Dimensional Flow Around a Propeller," AIAA Paper 82-0957.

[9] Jameson, A., and Caughy, D. A., " A Finite Volume Method for Transonic Potential Flow Calculations," Proceedings Third AIAA Computational Fluid Dynamics Conference, Albuquerque, 1977.

[10] Chausee, D. S.," Computation of Three-Dimensional Flow Through Prop Fans," Nielsen Engineering and Research Inc., NEAR TR-199, June 1979.

[11] Whitfeild, D. L., Swafford, T. W., Janus, J. M., Mulac, R. A., Belk, D. M., "Three-Dimensional Unsteady Euler Solutions for Propfans and CounterRotating Propfans in Transonic Flow," AIA A Paper 87-1197, June 1987.

[12] Matsuo, Y., Arakawa, C., Saito, S., and Kobayashi, H., "Navier-Stokes Computations for Flowfield of an Advanced Turboprop," AIAA Paper 883094 .

[13] Bober, L. A., Mitchell, G. A.," Summary of Advanced Methods for Predicting High Speed Propeller Performance," NASA TM 81409, 1980. 
[14] Lax, P. D., "Weak Solutions of Nonlinear Hyperbolic Equations and their Numerical Computation," Communications in Pure and Applied Mathematics, Vol. 7, pp. 159-193.

[15] Rizk, Y. M. and Chaussee, D. S., "Three-Dimensional Viscous-Flow Computations Using a Directionally Hybrid Implicit-Explicit Procedure," AIAA paper 83-1910, 1983.

[16] Jameson, A., Schimdt, W. and Turkel, E., "Numerical Solutions of the Euler Equations by Finite Volume Methods Using Runge-Kutta Time-Stepping Schemes," AIAA Paper 81-1259, 1981.

[17] Kaza, K. R. V., et al.," Analytical Flutter Investigation of a Composite Propfan Model," Journal of Aircraft, Vol. 26, No. 8, pp 772-780, A ugust 1989.

[18] Subrahmanyan, K. B., Kaza, K. R. V., Brown, G. V., and Lawrence, C.," Nonlinear Bending-Torsional Vibration and Stability of Rotating, Pretwisted, Preconed Blades Including Coriolis Effects," NASA TM 87207, 1986.

[19] "The NASTRAN Theoretical Manual," NASA SP 221(06), 1981.

[20] August, R., and Kaza, K. R. V.;" Vibration, Performance and Flutter Response Characteristics of a Large-Scale Propfan and its Aeroelastic Model," AIAA Paper 88-3155, 24 $4^{\text {th }}$. Joint Propulsion Conference, Boston, Massachusetts, July 11-13, 1988.

[21] Ruo, S. Y., and Sankar, L. N., "Euler Calculations for Wing-Alone Configuration," Journal of Aircraft, Vol. 25, No. 5, pp 436-441, May 1988.

[22] Wake, B. E., and Sankar, L. N., "Solutions of the Navier-Stokes Equations for the Flow about a Rotor Blade," Journal of the American Helicopter Society, Vol. 34, No. 2, pp 13-23, April 1989. 
[23] Rohrbach, C., Metzger, F. B., Black, D. M., and Ladden, R. M., "Evaluation of Wind Tunnel Performance Testings of an Advanced $45^{\circ}$ Swept EightBladed Propeller at Mach Numbers From 0.45 to 0.85," NASA CR-3505, 1982.

[24] Sullivan, W. E., Turnberg, J. E., and Violette, J. A.," Large Scale Advanced Propfan (LAP) Blade Design," NASA CR 174790.

[25] Aiello, R. A., and Chi, S.," Advanced Composite Turboprops: Modeling, Structural and Dynamic Analyses," ASME Paper 87-GT-78, 1987.

[26] Chou, S., "SR7L Turboprop Blade Finite Element Model," Sverdrup Topical Report, March 1986.

[27] Bushnell, P.," Measurement of the Steady Surface Pressure Distribution on a Single Rotation Large Scale Advanced Numbers from 0.03 to 0.78 ," NASA CR 182124, July 1988. 


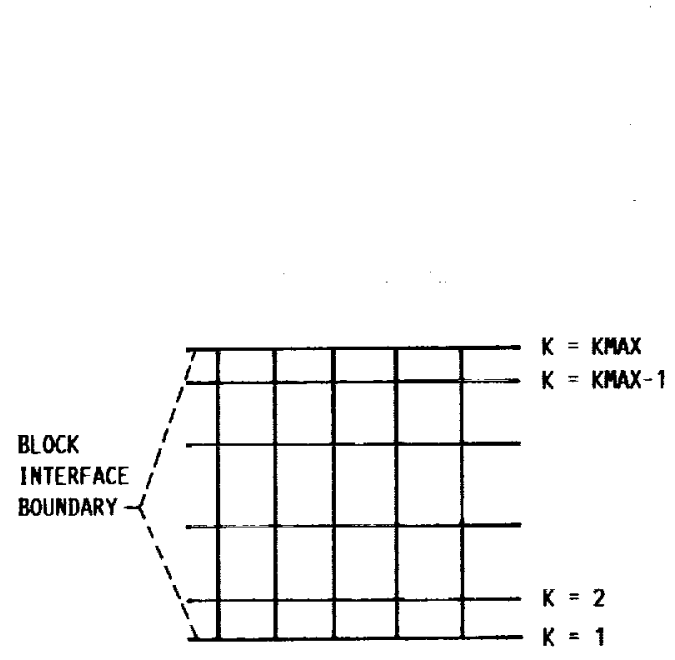

(a) AXISYMUETRIC.

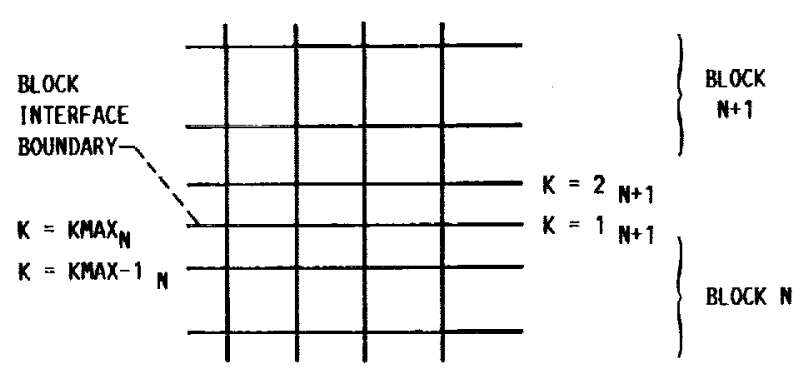

(b) ASYMMETRIC.

FI6. 1. BLOCK-INTERFACE BOUNDARIES.

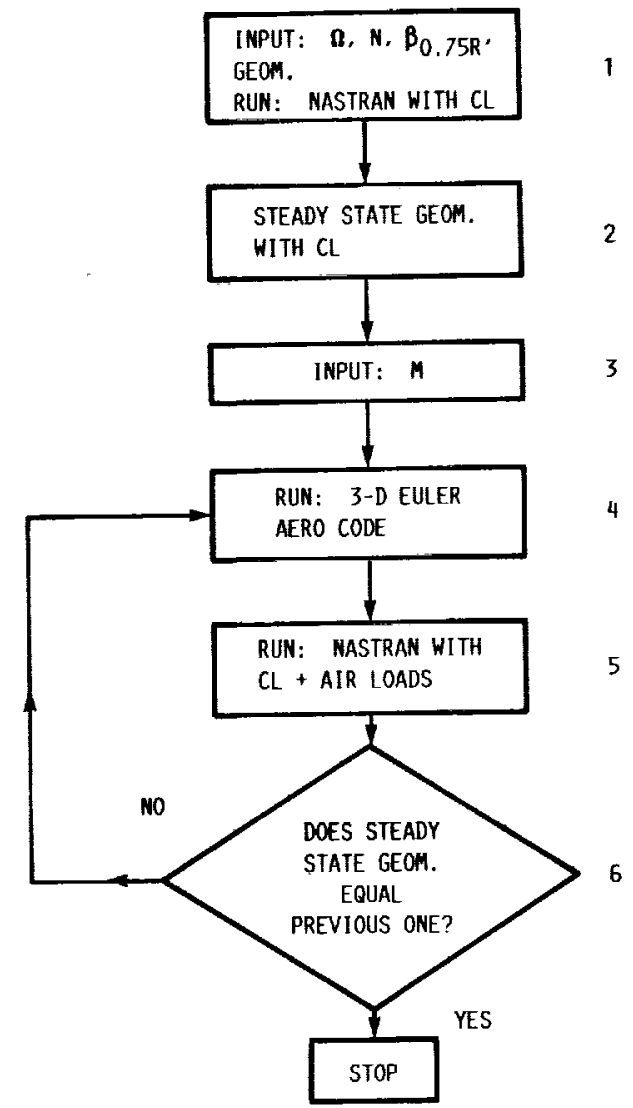

FIG, 2. FLOW CHART OF THE AEROELASTIC ANALYSIS.
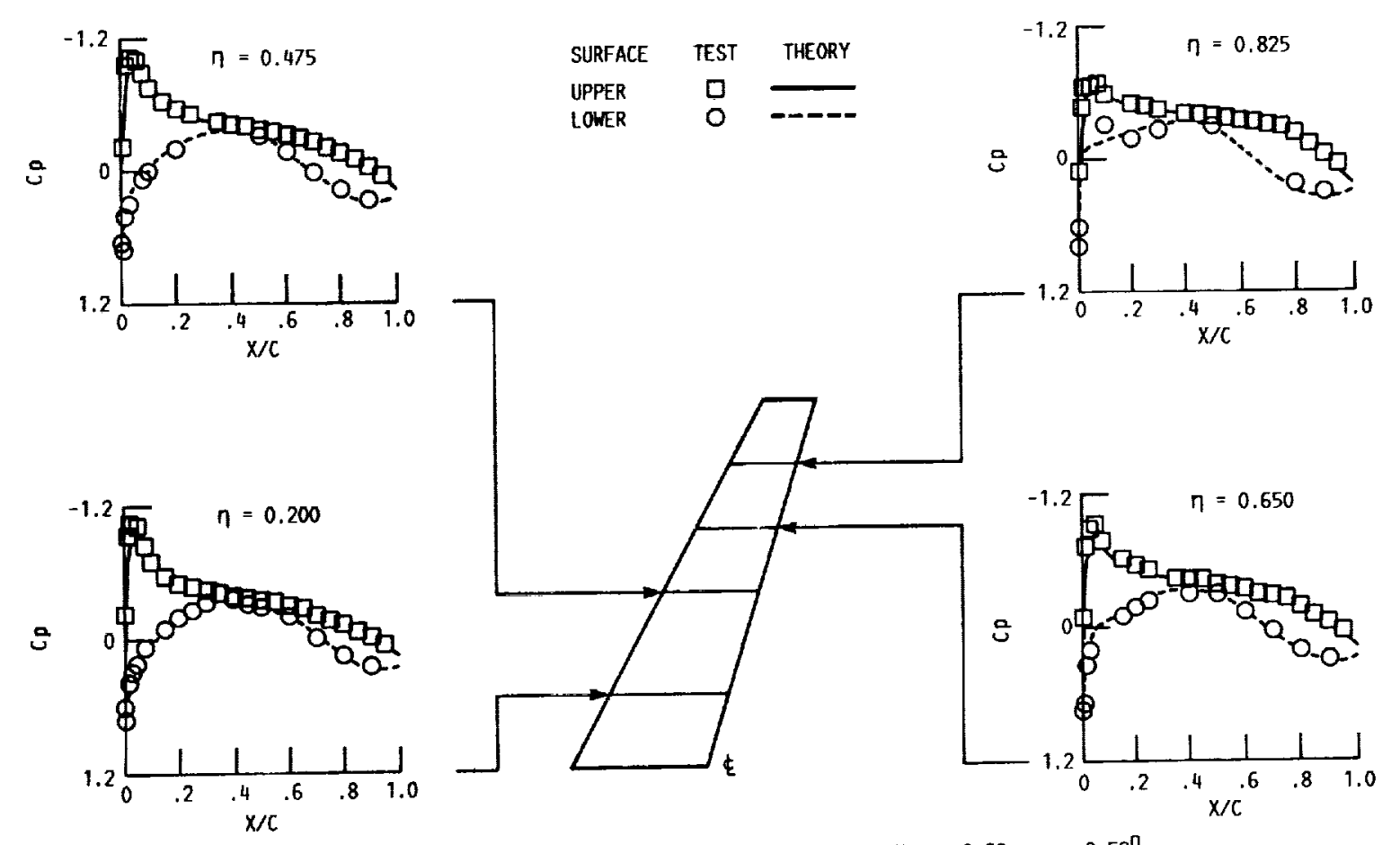

FI6. 3. EULER CALCULATIOHS FOR A NIMG (REF, 20), $M_{\infty}=0.62 ; a_{0}=0.58^{\circ}$. 


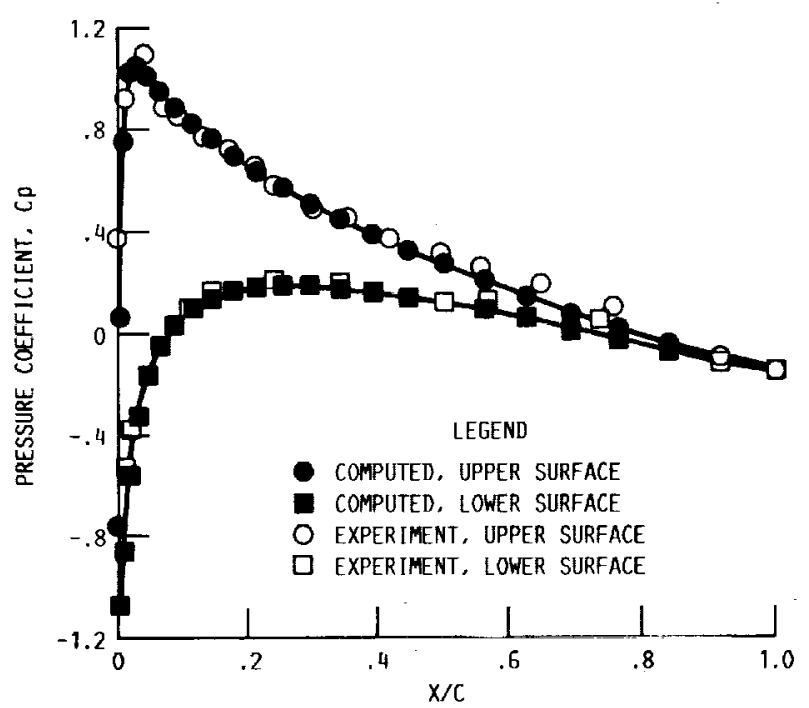

(a) PRESSURE DISTRIBUTION. $y / R=0,800$.

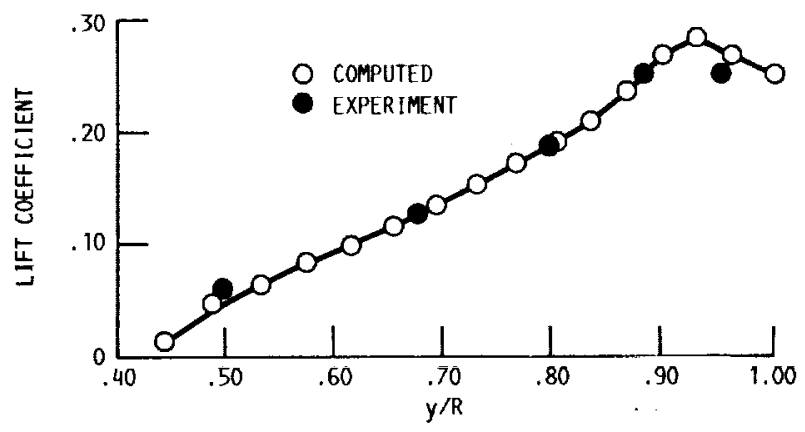

(b) LIFT DISTRIBUTION.

FIG. 4. DISTRIBUTION COEFFICIENTS FOR NACA 0012 ROTOR BLADE (FROM REF. 21).

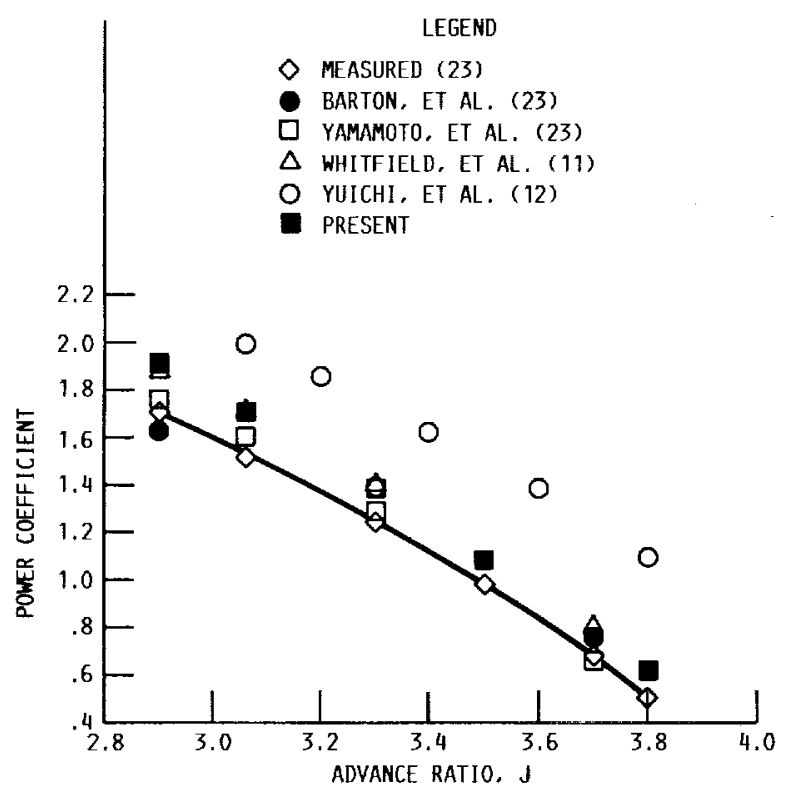

FIG, 6, POWER COEFFICIENT VERSUS ADVANCE RATIO FOR SR-3, 8-BLADED PROPFAN. $M_{\infty}=0.8$.

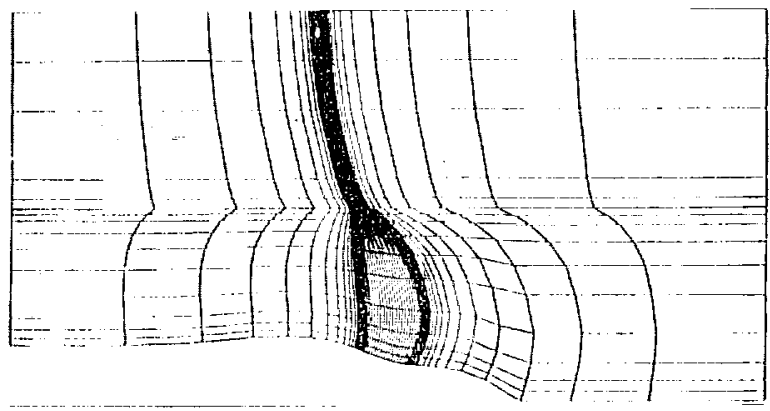

(a) H-GRID IN STREAMWISE PLANE. I $=1$ TO 60; $\mathrm{J}=1$ T0 16; $K=1$ TO 1 .

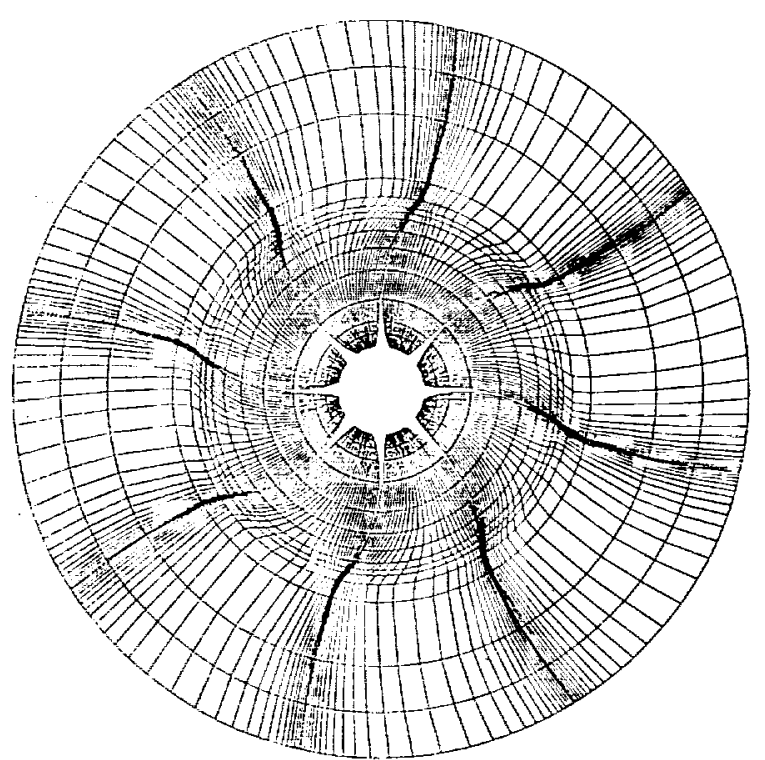

(b) O-GRID IN AZIMUTHAL PLANE. I $=30$ TO $30 ; \mathrm{J}=1$ TO 16 ; $K=1$ TO 29 .

FIG. 5. GRIDS.

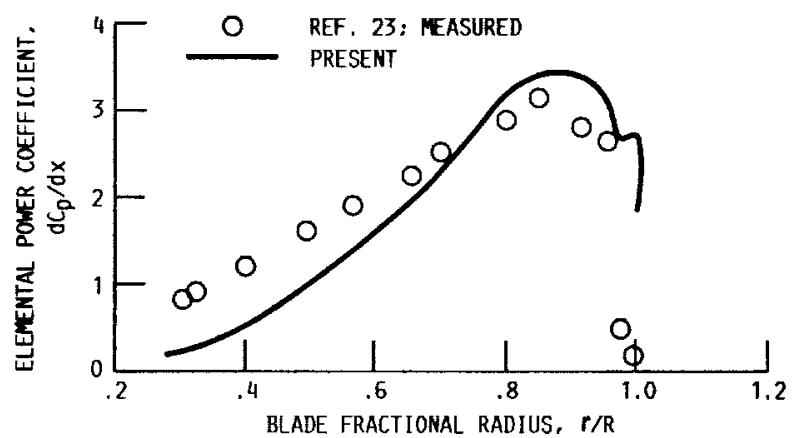

FI6. 7. ELEMENTAL POWER COEFFICIENT VERSUS RADIUS FOR SR-3, 8-BLADED PROPFAN. $J=3.002: M_{\infty}=0.8$; $\beta=60.5$. 


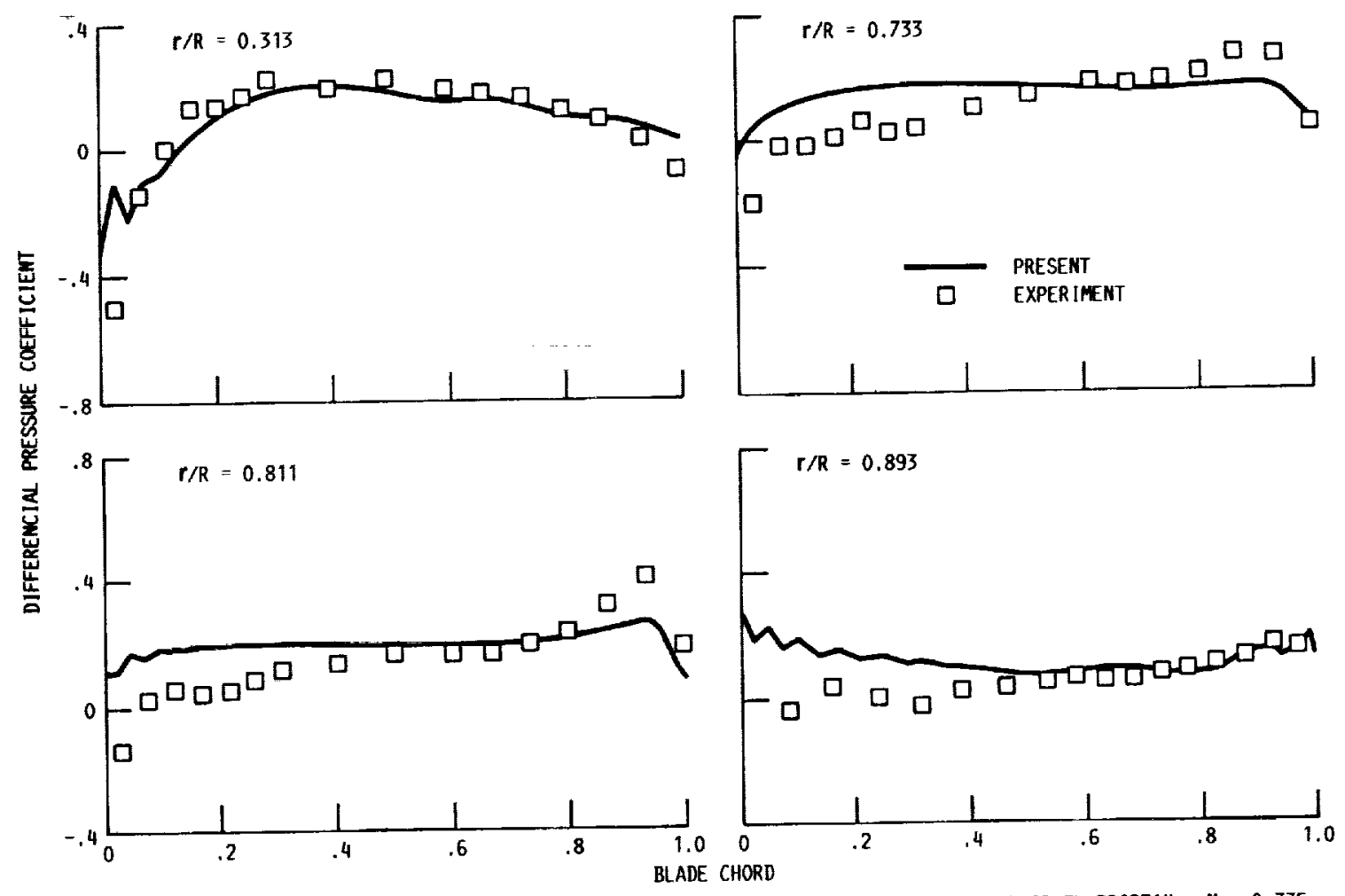

FIG. 8. PRESSURE COEFFICIENT DIFFERENCE AT VARIOUS RADIAL LOCATIONS FOR 2 BLADED SR-7L PROPFAN. M $=0,775$; $J=3.088 ; \beta=54.6^{\circ}$.

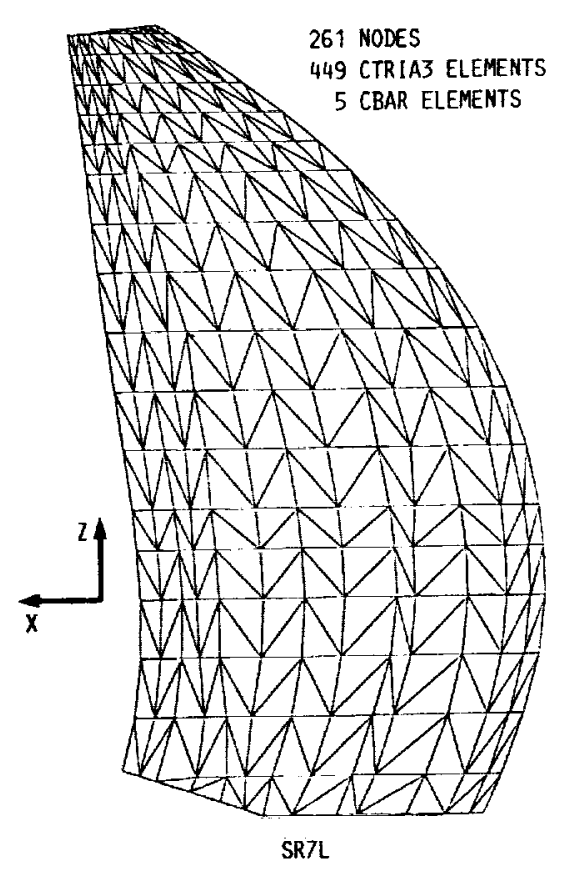

(a) PROPFAN BLADE FINITE-ELEMENT MODEL (REF. 19).

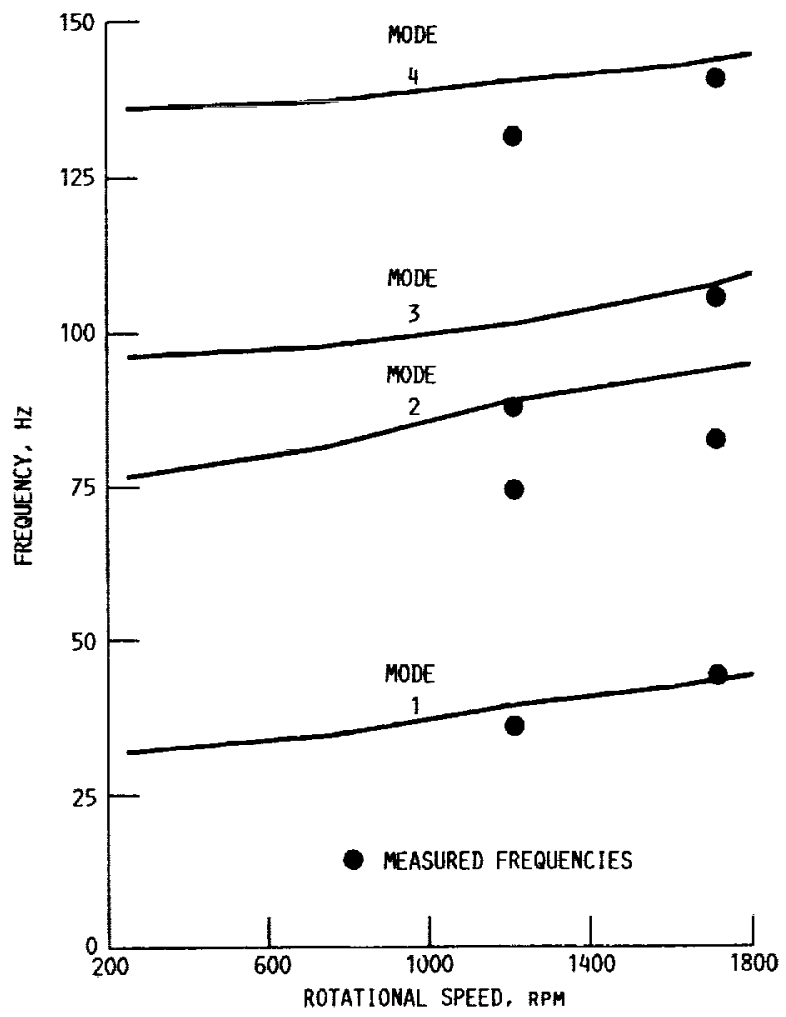

(b) SR-7L FREQUENCIES VERSUS RPM (REF. 19).

FIG. 9. SR-7L MODAL ANALYSIS. 


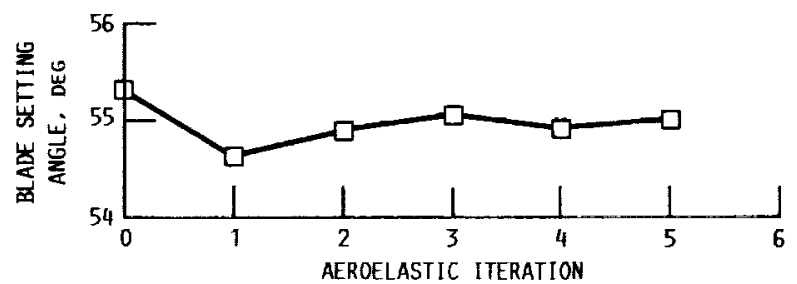

FIG, 10, BLADE SETTING ANGLE AT 75 PERCENT SPAN VERSUS AEROELASTIC ITERATION FOR SR-7L PROPFAN. $J=3.088$; $\mathrm{M}=0.775$.

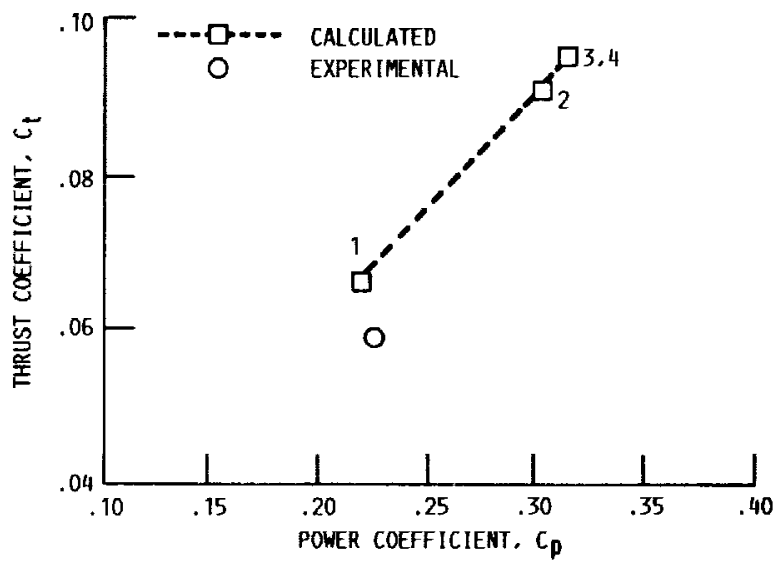

FIG. 11. CALCULATED THRUST COEFFICIENT VERSUS POMER COEFFICIENT FOR EACH AEROELASTIC ITERATION SR-7. 2-BLADED PROPFAN: $J=3.088 ; M=0.775$.

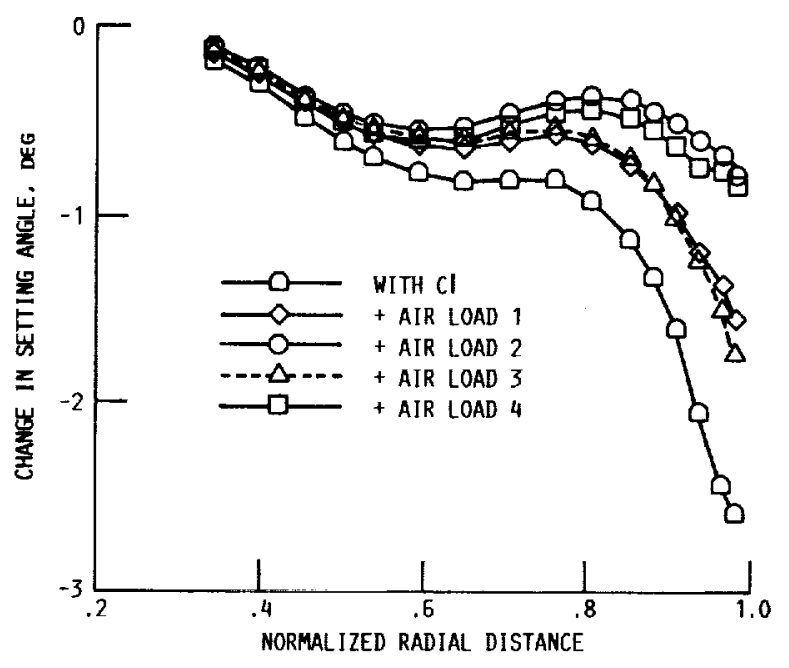

FIG, 12. CHANGE IN BLADE SETTING ANGLE VERSUS BLADE SPAN FOR SR-7L PROPFAN. $N=0.775 ; J=3.088$. 


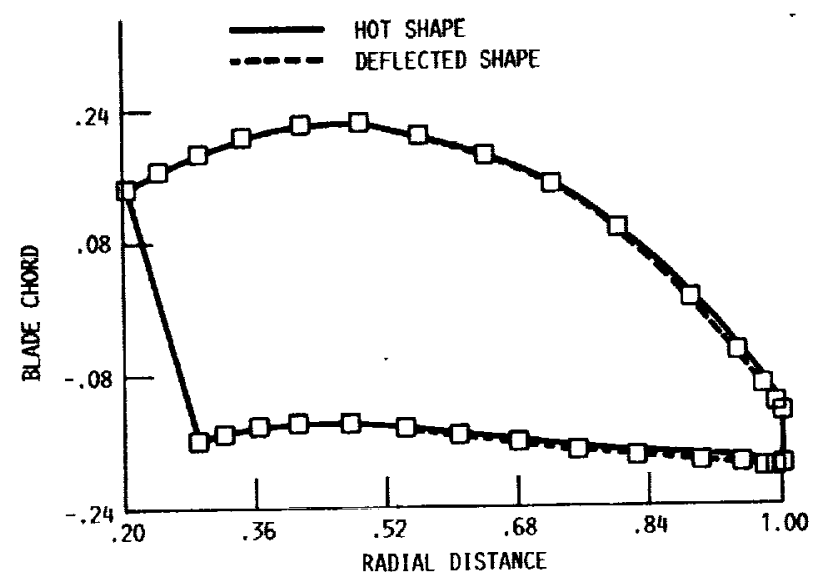

FIG. 13. IN PLAME DEFLECTION OF BLADE PLANFORM FOR SR-7L, 2 BLADED PROPFAN. $M=0.775 ; j=3.088$ : $B=54.6^{\circ}$.

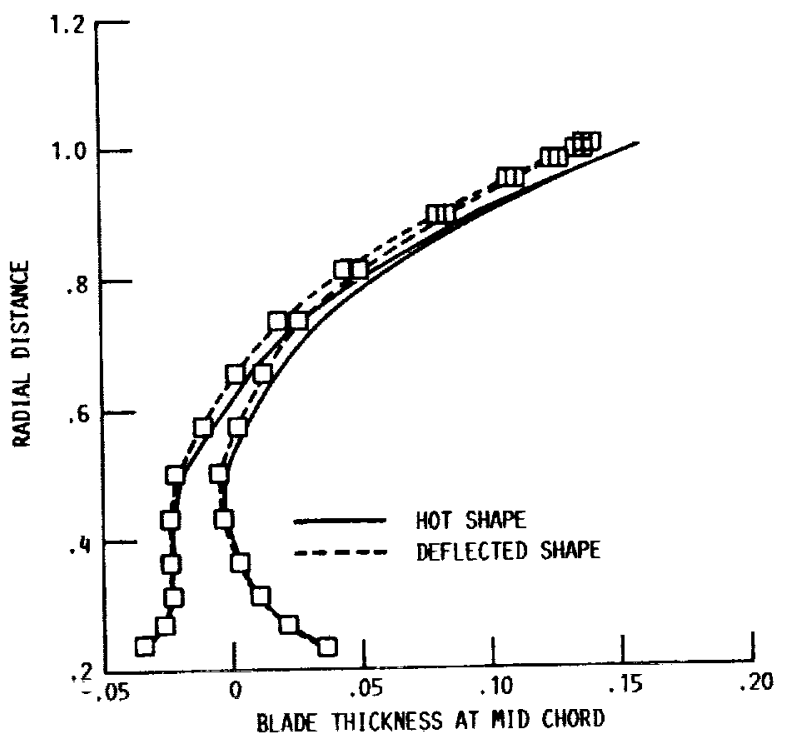

FIG. 14. OUT-OF-PLAME DEFLECTION AT HID CHORD FOR SR-7L, 2-BLADED PROPFAN. $M=0.775 ; J=3.088$; $B=54.6^{\circ}$. 


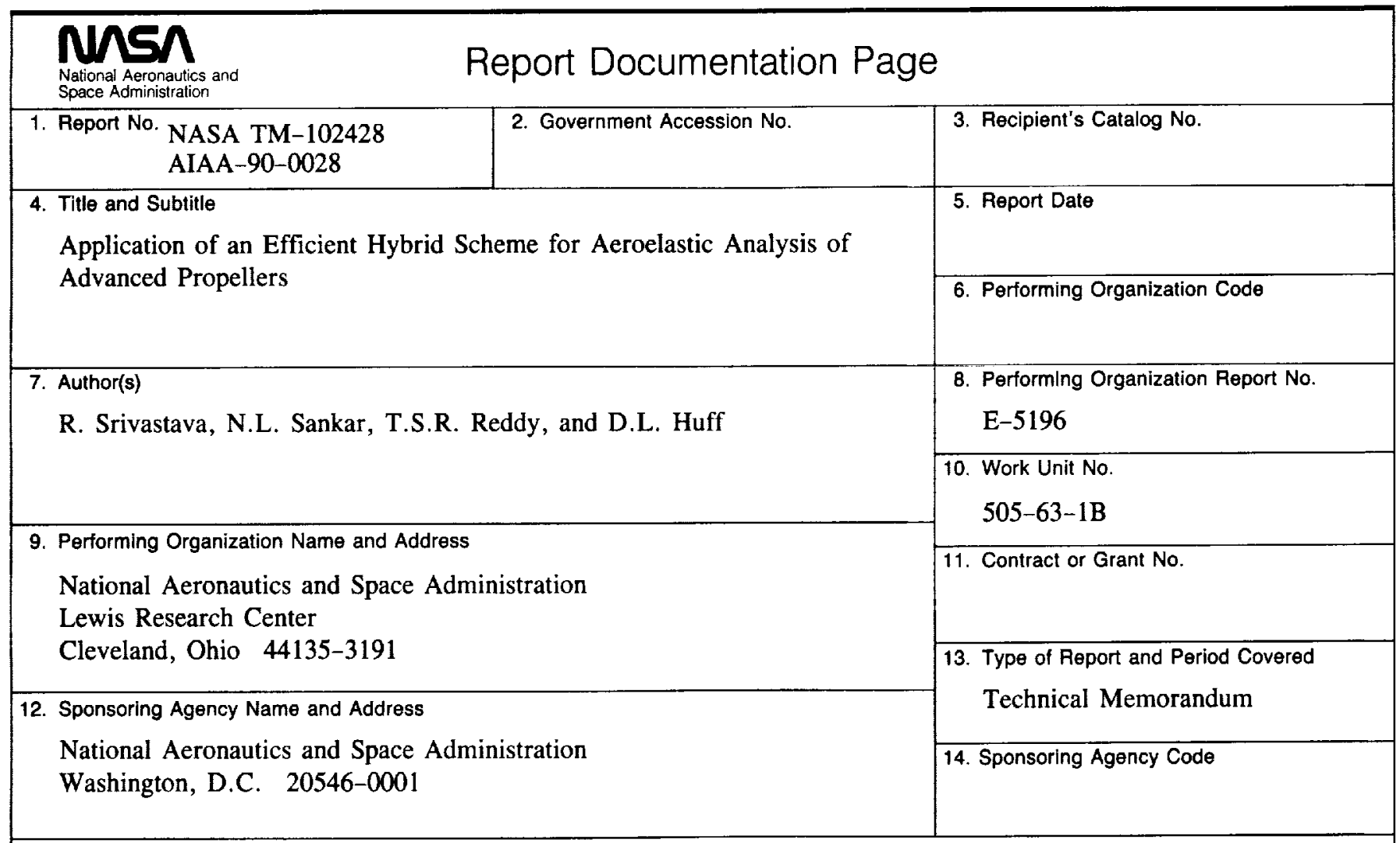

15. Supplementary Notes

Prepared for the 28th Aerospace Sciences Meeting sponsored by the American Institute of Aeronautics and Astronautics, Reno, Nevada, January 8-11, 1990. R. Srivastava and N.L. Sankar, Georgia Institute of Technology, Atlanta, Georgia; T.S.R. Reddy, The University of Toledo, Toledo, Ohio and NASA Resident Research Associate at Lewis Research Center; D.L. Huff, Lewis Research Center.

16. Abstract

An efficient three-dimensional hybrid scheme is applied for solving Euler equations to analyze advanced propellers. The scheme treats the spanwise direction semi explicitly and the other two directions implicitly, without affecting the accuracy, as compared to a fully implicit scheme. This leads to a reduction in computer time and memory requirement. The calculated power coefficients for two advanced propellers, SR3 and SR7L, and various advance ratios showed good correlation with experiment. Spanwise distribution of elemental power coefficient and steady pressure coefficient differences also showed good agreement with experiment. A study of the effect of structural flexibility on the performance of the advanced propellers showed that structural deformation due to centrifugal and aero loading should be included for better correlation.

17. Key Words (Suggested by Author(s))

Propfan; Euler; Hybrid scheme; Blade flexibility; NASTRAN; Performance
18. Distribution Statement

Unclassified-Unlimited

Subject Category 02

\begin{tabular}{|c|c|c|c|}
\hline $\begin{array}{c}\text { 19. Security Classif. (of this report) } \\
\text { Unclassified }\end{array}$ & $\begin{array}{c}\text { 20. Security Classif. (of this page) } \\
\text { Unclassified }\end{array}$ & $\begin{array}{c}21 . \text { No of pages } \\
30\end{array}$ & $\begin{array}{c}22 . \text { Price* } \\
\text { A03 }\end{array}$ \\
\hline
\end{tabular}


